\title{
Fitotoxicidade do óleo essencial de Mikania Laevigata sobre o desenvolvimento inicial de duas espécies invasoras
}

Produtos naturais tem se mostrado eficazes no controle de plantas invasoras em áreas de produção agrícola. Dentre esses, os óleos essenciais destacam-se por serem bons agentes alelopáticos. Objetivou-se avaliar a fitotoxicidade do óleo essencial de Mikania laevigata no crescimento inicial das plantas invasivas Bidens pilosa e digitaria insularis. O óleo essencial foi extraído pelo método da hidrodestilação e seus componentes foram analisados por CG-EM. A inibição do crescimento inicial das duas espécies invasoras utilizadas neste estudo foi avaliada utilizando duas concentrações de óleo essencial (0,5 e 1\%). Os parâmetros analisados foram comprimentos das raízes e das partes aéreas das plântulas. Na ocorrência de efeitos significativos, os dados obtidos foram submetidos à análise de médias, as quais foram comparadas pelo teste de Tukey, com $5 \%$ de significância. Germacreno D $(41,6 \%)$ foi o principal constituinte químico encontrado no óleo essencial. Para as duas espécies invasoras testadas, a concentração do óleo essencial a $1 \%$ foi a que apresentou melhores resultados para os parâmetros analisados.

Palavras-chave: Guaco; Alelopatia; Bidens Pilosa; Digitaria Insularis.

\section{Phytoxicity of Mikania Laevigata essential oil on the initial development of two invasive species}

\begin{abstract}
Natural products have been proven effective in controlling invasive plants in agricultural production areas. Among these, the essential oils stand out as being good allelopathic agents. The aim of this study was to evaluate the essential oil phytotoxicity of M. laevigata on the initial growth of the invasive plants Bidens pilosa and digitaria insularis. The essential oil was extracted by the hydrodistillation method and its components were analyzed by GC-MS. The Inhibition of initial growth of the two invasive species used at this study was evaluated using two concentration of the essential oil (0.5 and 1\%). The following parameters were analyzed: lengths of the roots and aerial parts of the seedlings. When the effects presents significants, the data were submitted to the analysis of the means, which were compared by the Tukey test, with $5 \%$ significance. Germacrene D $(41.6 \%)$ was the main chemical constituent found in essential oil. For the two invasive species tested, the concentration of essential oil at $1 \%$ was the one that presented the best results for the parameters analyzed.
\end{abstract}

Keywords: Guaco; Allelopathy; Bidens Pilosa; Digitaria Insularis.

Topic: Proteção de Plantas e Fitotecnia

Reviewed anonymously in the process of blind peer.

Livia Maria de Lima Santos

Universidade Federal de Lavras, Brasil

http://lattes.cnpq.br/2462594377000843

livia-linne@hotmail.com

Carolina Mesquita Germano

Universidade Federal de Lavras, Brasil

http://lattes.cnpq.br/5740745559516003

carolmgermano@gmail.com

Adenilson Henrique Gonçalves

Universidade Federal de Lavras, Brasil

http://lattes.cnpq.br/8053622224381109

adenilsonhg@dag.ufla.br

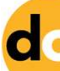

DOI: 10.6008/SPC2179-6858.2017.004.0001
Received: 01/07/2017

Approved: 01/10/2017

\author{
Suzan Kelly Vilela Bertolucci \\ Universidade Federal de Minas Gerais, Brasil \\ http://lattes.cnpq.br/7307740195142206 \\ suzan@dag.ufla.br
}

Referencing this:

SANTOS, L. M. L.; GERMANO, C. M.; GONÇALVES, A. H.; BERTOLUCCI, S. K. V.. Fitotoxicidade do óleo essencial de Mikania Laevigata sobre o desenvolvimento inicial de duas espécies invasoras. Revista lberoAmericana de Ciências Ambientais, v.8, n.4, p.1-7, 2017. DOI: http://doi.org/10.6008/SPC2179-6858.2017.004.0001 


\section{INTRODUÇÃO}

O uso de herbicidas sintéticos para combater as plantas invasoras de áreas cultivadas chega a 352,3 mil toneladas por safra no Brasil (CASSAL, 2014), e embora tenham sucesso no controle das plantas invasoras, podem causar danos à saúde humana e ao ambiente, além de promover o desenvolvimento de plantas resistentes aos herbicidas. Foram identificados em todo o mundo 249 plantas invasoras resistentes aos herbicidas (144 dicotiledóneas e 105 Monocotiledôneas) (HEAP 2014; ISMAIL et al., 2016).

Neste contexto, os produtos naturais estão ganhando atenção devido ao controle eficaz das plantas invasoras e suas propriedades não poluentes, como rápida degradação no ambiente e baixa toxicidade para os animais (DAYAN et al., 2012). Entre os diferentes produtos vegetais naturais, os óleos essenciais se destacam por serem poderosos inibidores da germinação de sementes e promoverem redução do crescimento inicial de plantas invasoras (AYEB-ZAKHAMA et al., 2017; ISMAIL et al., 2016). Podendo causar diversas alterações morfofisiológicas, como a degradação das membranas celulares e a desestruturação dos microtúbulos (CHAIMOVITSH et al., 2017)

Os óleos essenciais, de maneira geral, são compostos por misturas complexas de substancias voláteis, caracterizados por um forte odor e sintetizados via metabolismo secundário das plantas. Seus principais constituintes são os fenilpropanoides e terpenoides (ANDRADE et al., 2012). Mikania laevigata Schultz Bip. ex Baker, conhecida como guaco, é nativa da Floresta Atlântica brasileira e suas folhas são amplamente utilizadas em afecções do trato respiratório devido ao elevado teor de cumarina (1,2-benzopirona), incluída na Farmacopeia Brasileira desde 2005 (BRANDÃO et al., 2006). O óleo essencial do gênero Mikania é rico em sesquiterpenos e diterpenos (SILVA JÚNIOR et al., 1994).

Os sesquiterpenos são descritos como possuidores de variada atividade biológica, como antialérgica (GHELARDINI et al., 2001), anestésica local (COSTA et al., 2000), antifúngica (ZHENG et al., 1992), anticarcinogênica (CHINOU et al., 1996) e diminuição do crescimento de plantas invasoras de áreas cultivadas (AYEB-ZAKHAMA et al., 2017; VERDEGUER et al., 2011).

Bidens pilosa L. (Asteraceae), conhecida popularmente como picão, é originária da América tropical, sendo uma das plantas invasoras de maior dispersão e dificuldade de controle em áreas agricultáveis (SANTOS et al., 2011). Digitaria insularis (L.) Mez. ex Ekman. (Poaceae), conhecida popularmente como capim-amargoso, é considerada invasora em mais de 60 países, ocorrendo em mais de 30 culturas de importância econômica (MONDO et al., 2010). Objetivou-se avaliar a fitotoxiciade do óleo essencial de $M$. laevigata sobre o crescimento inicial de B. pilosa e D. insularis, com vistas a propor a sua utilização no controle de plantas invasoras em áreas de produção agrícola.

\section{MATERIAL E MÉTODOS}

\section{Material vegetal e extração do óleo essencial}

M. laevigata (guaco) foi cultivada no Horto de Plantas Medicinais da Universidade Federal de Lavras. Amostra da espécie foi depositada no Herbário ESAL, sob o registro de numeração 57818. Folhas frescas, sadias e homogêneas no período da manhã, sem precipitação pluvial, foram coletadas e secas a $40^{\circ} \mathrm{C}$ em 
estufa com circulação forçada de ar. Após a secagem foram picadas $(121,6 \mathrm{~g})$ e a hidrodestilação, em triplicata, utilizando um aparelho de Clevenger modificado, por duas horas. Os óleos foram separados por decantação, armazenados em frascos âmbar com capacidade de $2 \mathrm{~mL}$ e mantidos sob refrigeração $\left(4^{\circ} \mathrm{C}\right)$, até as análises químicas. $O$ teor do óleo essencial foi determinado e expresso em g.100g- ${ }^{1}$ de matéria seca das folhas.

\section{Análises químicas do óleo essencial}

As análises quantitativas foram feitas por cromatografia em fase gasosa acoplado a um detector de ionização em chama de hidrogênio (CG-DIC) em um sistema Agilent ${ }^{\circledR} 7890 \mathrm{~A}$, equipado com coluna capilar DB-WAX $(30 \mathrm{~m} \times 250 \mu \mathrm{m} \times 0,25 \mu \mathrm{m})$, utilizou-se hélio como gás de arraste com fluxo de 1,0mL/min; as temperaturas do injetor e do detector foram mantidas a $250^{\circ} \mathrm{C}$.

O óleo foi diluído em acetato de etila $(1 \%, v / v)$ e injetado automaticamente no cromatógrafo empregando volume de injeção de 1,0 $\mu \mathrm{L}$, no modo split a uma razão de injeção de 50:1. A separação dos constituintes voláteis foi realizada em uma rampa de temperatura de $3^{\circ} \mathrm{C} / \mathrm{min}$ de $60^{\circ} \mathrm{C}$ a $230^{\circ} \mathrm{C}$, seguida de uma rampa de temperatura de $10^{\circ} \mathrm{C} / \mathrm{min}$ até $240^{\circ} \mathrm{C}$, mantendo uma condição isotérmica por um minuto.

As análises foram realizadas em triplicata e os resultados expressos pela média da porcentagem de área normalizada relativa dos picos cromatográficos \pm o desvio padrão. A avaliação qualitativa do óleo essencial foi realizada em Cromatógrafo Agilent ${ }^{\circledR} 7890 A$ acoplado a um detector seletivo de massas Agilent ${ }^{\circledR}$ MSD 5975C (Agilent Technologies, Califórnia, EUA), operado por ionização de impacto eletrônico a 70 e V, em modo varredura, a uma velocidade de $1,0 \mathrm{scan} / \mathrm{s}$, com intervalo de aquisição de massas de $40-400 \mathrm{~m} / \mathrm{z}$. As condições operacionais foram as mesmas empregadas nas análises por CG-DIC.

Os constituintes químicos foram identificados por comparação dos seus índices de retenção relativos à coinjeção de uma solução padrão de $n$-alcanos (C8-C20, Sigma-Aldrich ${ }^{\circledR}$, St. Louis, USA) e por comparação aos espectros de massa do banco de dados da biblioteca NIST/EPA/NHI (NATIONAL INSTITUTE OF STANDARDS AND TECHNOLOGY - NIST, 2008) e da literatura (ADAMS, 2007). Os índices de retenção foram calculados com base na equação de Van den Dool e Kratz e para as atribuições foram consultados índices de retenção citados em literatura (ADAMS, 2007).

\section{Ensaio fitotóxico}

Uma solução 'mãe' do óleo essencial foi preparada, a partir de 0,5g do óleo, utilizando Tween 80, na proporção 1:1 (v/v) e dissolvida em água destilada (q.s.p. 100mL de solução). A partir desta preparam-se as soluções testes nas concentrações finais 1 e 0,5\%. Como controle foi utilizado uma solução de Tween 80 a 1,0\% v/v e água (SILVA et al., 2005). As soluções testadas foram adicionadas apenas no início dos bioensaios, e posteriormente, quando necessário, apenas água destilada foi acrescentada.

Foram utilizadas 10 plântulas com três dias de idade (72h) em cada caixa acrílica, tipo gerbox (dimensões $11 \times 11 \times 4 \mathrm{~cm}$ ), tendo como substrato duas folhas de papel mata-borrão esterilizadas a $120^{\circ} \mathrm{C}$ por 
30min. Quatro caixas por tratamento, totalizando 40 plântulas que foram mantidas em câmara de crescimento (BOD) à temperatura de $26 \pm 1^{\circ} \mathrm{C}$ e fotoperíodo de $8 / 16 \mathrm{~h}$.

Após 10 dias de crescimento, os comprimentos das raízes e das partes aéreas das plântulas foram realizados, com auxílio de um paquímetro digital. Os resultados foram expressos em milímetros. A determinação da biomassa seca total das plântulas foi realizada em estufa a $70^{\circ} \mathrm{C}$, em embalagens de papel Kraft, até obtenção das massas constantes. Depois desse período, efetuou-se a pesagem e determinação das médias por repetição, sendo os resultados expressos em miligramas. O delineamento experimental utilizado foi inteiramente casualizado em esquema fatorial $2 \times 2$ (concentrações do óleo essencial $x$ espécies), com quatro repetições. Na ocorrência de efeitos significativos, as médias foram comparadas pelo teste de Tukey, com $5 \%$ de significância.

\section{RESULTADOS E DISCUSSÃO}

O teor do óleo essencial de $M$. laevigata foi de $0,092 \%$, superior quando comparado aos relatos de Duarte et al. (2005), que obtiveram 0,05\% de óleo essencial a partir de $40 \mathrm{~g}$ de folhas frescas de M. laevigata. O óleo essencial de $M$. laevigata constituiu de $82,75 \%$ de sesquiterpenos, representados por cinco constituintes químicos (germacreno $D$, biciclogermacreno, espatulenol, $\beta$-cariofileno e óxido de cariofileno). Cerca de $50 \%$ do teor de sesquiterpenos totais foi atribuído ao germacreno D (41,6\%). Rehder et al. (2006) também encontraram que o principal sesquiterpeno presente no óleo essencial extraído das folhas de $M$. laevigata e M. glomerata foi o germacreno D, com $23,6 \%$ e $23,4 \%$ respectivamente.

Tabela 1: Composição química do óleo essencial das folhas secas de Mikania laevigata.

\begin{tabular}{|c|c|c|}
\hline IR & Constituintes & Área (\%) \\
\hline 1484 & Germacreno D & 41,6 \\
\hline 1499 & Biciclogermacreno & 17,49 \\
\hline 1580 & Espatulenol & 10,89 \\
\hline 1421 & $\beta$-cariofileno & 9,02 \\
\hline 1586 & Óxido de cariofileno & 3,75 \\
\hline & Total (\%) & 82,75 \\
\hline
\end{tabular}

Legenda: Índice de retenção relativo à série $n$-alcanos (C8 - C20) em coluna DB-WAX na ordem de eluição.

Na avaliação dos efeitos fitotóxicos, observou-se variação em função da concentração e da planta receptora $(D$. insularis e $B$. pilosa). O óleo essencial de $M$. laevigata na concentração de $1 \%$ inibiu o crescimento radicular $(54,77 \%)$, do hipocótilo $(43,36 \%)$, da biomassa seca total (21\%) (Tabela 2 ) e da expansão da área foliar $(47,4 \%)$ nas plântulas de $D$. insularis em relação à testemunha (Figura 1).

Enquanto a $0,5 \%$ do óleo a radícula de $D$. insularis não foi sensível, mantendo-se com valores similares aos observados no controle, somente o hipocótilo reduziu $24,22 \%$ do comprimento. Para B. pilosa a concentração do óleo a 0,5\% provocou reduções de 23,19\% na radícula, 27,86\% no hipocótilo (Tabela 2) e 79,87\% na área foliar, ilustrada na figura 1, entretanto a $1 \%$ as reduções foram de $58,41 \%$ da radícula, 54,34\% do hipocótilo, $18 \%$ da massa seca e $87,73 \%$ da área foliar, em relação à testemunha.

O parâmetro biométrico mais sensível para detectar a bioatividade do óleo frente às duas espécies invasoras testadas foi à redução da área foliar. Pode ser observado na figura 2, nas imagens b e c, que o óleo 
de $M$. laevigata também provocou em $D$. insularis clorose intensa. Esse fato pode estar relacionado à presença do B-cariofileno $(9,02 \%)$ no óleo essencial. Segundo Sánchez-Muñoz et al., (2012), o B-cariofileno foi capaz de inibir a fitoeno desaturase em Physalis ixocarpa, uma enzima chave na biossíntese dos carotenoides. É sabido que os carotenoides protegem a clorofila da foto-oxidação, e sua carência resultaria em perda da clorofila e clorose evidente, podendo levar a planta a morte. Vários herbicidas que atuam no FSII, como o Paraquat, atua também induzindo a degradação da clorofila, bem como outros mecanismos de ação (SANTOS et al., 1999).

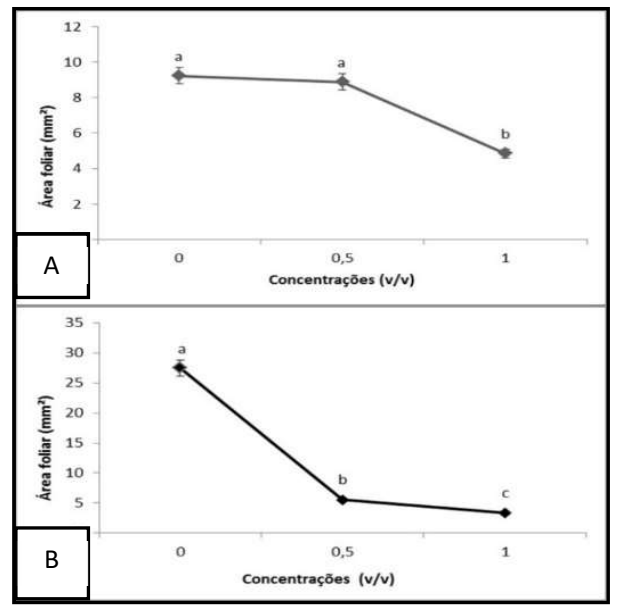

Figura 1. Área foliar de Digitaria insularis (A); e Bidens pilosa (B) submetidas a diferentes concentrações do óleo essencial de Mikania laevigata. As médias seguidas das mesmas letras não diferem estatisticamente entre si, pelo teste de Tukey a $5 \%$ de probabilidade.

As radículas das plântulas foram mais sensíveis se comparado ao hipocótilo (Figura 2), principalmente na espécie $B$. pilosa, onde foi observado o encurvamento das raízes $(1 \% \mathrm{v} / \mathrm{v})$ e pontos de necrose, ou seja, locais onde ocorreu morte do tecido. Zhu et al. (2016), ao testar o óleo essencial de Scutellaria strigillosa, que possui como componente majoritário o germacreno $D$, também verificaram inibição do crescimento da radícula, no entanto de forma mais expressiva (86\%). Segundo Scrivanti (2010), as raízes são mais sensíveis aos metabólitos secundários, pois estão em contato direto com o substrato.

Os resultados do presente estudo indicam que o óleo essencial de M. laevigata possui atividade bioherbicida, embora para melhor caracterização da atividade fitotóxica seja necessário conduzir experimentos adicionais em casa de vegetação e campo. Bem como determinar os principais modos de ação, ou seja, quais são os sítios de ação (alvo molecular) onde o óleo irá atuar na planta receptora.

Tabela 2: Comprimento do hipocótilo $(\mathrm{mm})$; Comprimento da radícula ( $\mathrm{mm}$ ) e Massa seca (mg) das espécies Digitaria insularis e Bidens pilosa, submetidas ao óleo essencial de Mikania laevigata nas concentrações 0, 05 e $1 \%$ (v/v). Médias seguidas das mesmas letras não diferem estatisticamente entre si, pelo teste de Tukey a $5 \%$ de probabilidade.

\begin{tabular}{|c|c|c|c|}
\hline Concentração do OE (v/v) & Radícula (mm) & Hipocótilo (mm) & Massa seca (mg) \\
\hline \multicolumn{4}{|c|}{ Digitaria insularis } \\
\hline 0 & $42^{a}$ & $2,56^{a}$ & $0,0055^{a}$ \\
\hline $0,5 \%$ & $39^{a}$ & $1,94^{b}$ & $0,0054^{a}$ \\
\hline $1 \%$ & $19^{b}$ & $1,45^{c}$ & $0,0043^{b}$ \\
\hline CV \% & 20 & 18 & 14 \\
\hline \multicolumn{4}{|c|}{ Bidens pilosa } \\
\hline 0 & $44^{a}$ & $21,9^{a}$ & $0,0053^{a}$ \\
\hline $0,5 \%$ & $33,8^{b}$ & $15,8^{b}$ & $0,0052^{a}$ \\
\hline $1 \%$ & $18,3^{c}$ & $10^{c}$ & $0,0043^{b}$ \\
\hline C.V.(\%) & 22 & 19 & 7 \\
\hline
\end{tabular}




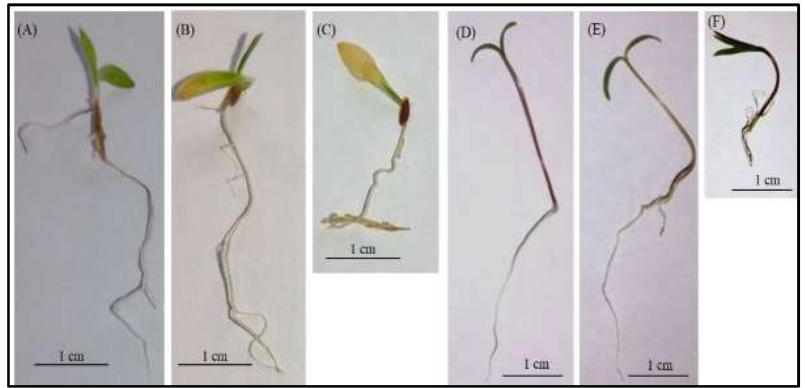

Figura 2: Fitotoxicidade de plântulas de duas espécies invasoras de áreas cultivadas submetidas ao óleo de Mikania laevigata. Digitaria insularis; (A) controle; (B) $0,5 \%$ do óleo essencial; (C) $1 \%$ do óleo essencial; Bidens pilosa; (D) controle; (E) 0,5\% do óleo essencial; (F) 1\% do óleo essencial.

\section{CONCLUSÃO}

O óleo essencial de $M$. laevigata na concentração de $1 \%$ apresentou potencial herbicida in vitro, afetando o crescimento inicial, principalmente radícula e área foliar de B. pilosa e $D$. insularis. E clorose, em D. insularis.

\section{REFERÊNCIAS}

ADAMS, R. P.. Identification of essencial oils componentes by gas chromatography/mass spectroscopy. 4 ed. Carol Stream: Allured, 2007.

ANDRADE, M. A.; CARDOSO, M. G.; BATISTA, L. R.; MALLET, A. C. T.; MACHADO, S. M. F.. Óleos essenciais de Cymbopogon nardus, Cinnamomum zeylanicum e Zingiber officinale: composição, atividades antioxidante e antibacteriana. Revista Ciência Agronômica, Fortaleza, v.43, p.399-408, 2012.

AYEB-ZAKHAMA, A. E.; SAKKA-ROUIS, L.; FLAMINI, G.; JANNET, H. B.; HARZALLAH-SKHIRI, F.. Chemical Composition and Allelopathic Potential of Essential Oils from Citharexylum spinosum L. Grown in Tunisia. Chemistry and Biodiversity, v.14, n.4, 2017. DOI:

http://doi.org.0.1002/cbdv.201600225

BRANDÃO, M. G. L.; COSENZA, G. P.; MOREIRA, R. A.; MONTE-MOR, R. L. M.. Medicinal plants and other botanical products from the Brazilian Offi cial Pharmacopoeia. Revista Brasileira de Farmacognosia, João Pessoa, v.16, p.408-420, 2006. DOI: http://doi.org.10.1590/S0102$\underline{695 \times 2006000300020}$

CASSAL, V. B.; AZEVEDO, L. F.; FERREIRA, R. P.; SILVA, D. G.; SIMÃO, R. S.. Agrotóxicos: uma revisão de suas consequências para a saúde pública. Revista Eletrônica em Gestão, Educação e Tecnologia Ambiental, Santa Maria, v.18, n.1, p.437-445, 2014.

CHAIMOVITSH, D.; SHACHTER, A.; ABU-ABIED, M.; RUBIN, B.; SADOT, E.; DUDAl, N.. Herbicidal Activity of Monoterpenes Is Associated with Disruption of Microtubule Functionality and Membrane Integrity. Weed Science, v.65, p.19-30, 2017. DOI: http://doi.org.10.1614/WS-D-16-00044.1

CHINOU, I. B.; ROUSSIS, V.; PERDETZOGLOU, D.; LOUKIS, A.. Chemical and biological studies on two Helichrysum species of Greek origin. Planta Medica, Copenhague, v.62, p.377379, 1996. DOI: http://doi.org.10.1055/s-2006-957914
COSTA, T. R.; FERNANDES, O. F. L.; SANTOS, S. C.; OLIVEIRA, C. M. A.; LIÃO, L. M.; FERRI, P. H.; PAULA, J. R.; FERREIRA, H. D.; SALES, B. H. N.; SILVA, M. R. R.. Antifungal activity of volatile constituents of Eugenia dysenterica leaf oil. Journal of Ethnopharmacology, Goiânia, v.72, p.111-117, 2000. DOI: http://doi.org.10.1016/S0378-8741(00)00214-2

DAYAN, F. E.; OWENS, D. K.; DUKE. S. O.. Rationale for a natural products approach to herbicide discovery. Pest Management Science, v.68, p.519-528, 2012. DOI: http://doi.org.10.1002/ps.2332

DUARTE, M. C. T.; FIGUEIRA, G. M.; SARTORATTO, A.; REHDER, V. L. G.; DELARMELINA, C.. Anti-Candida activity of Brazilian medicinal plants. Journal of Ethnopharmacology, Campinas, v.97, p.305-311, 2005. DOI: http://doi.org.10.1016/j.jep.2004.11.016

GHELARDINI, C.; GALEOTTI, N.; DI CESARE, M. L.; MAZZANTI, G.; BARTOLINI, A.. Local anaesthetic activity of betacaryophyllene. II Farmaco, v.56, p.387-389, 2001. DOI: http://doi.org.10.1016/S0014-827X(01)01092-8

HEAP, I.. Global perspective of herbicide-resistant wheat. Pest Management Science, v.70, p.1306-1315, 2014. DOI: http://doi.org.10.1002/ps.3696

ISMAIL, B. S.; HALIMSHAH, S.; WAN, J. W. A.; YUSOFF, N.. Allelopathic Potential of the Leaf and Seed of Pueraria javanica Benth. on the Germination and Growth of Three Selected Weed Species. Sains Malaysiana, v.4, n.45, p.517521, 2016. 
MONDO, V. H. Z.; CARVALHO, S. J. P.; DIAS, A. C. R.; MARCOS FILHO, J.. Efeitos da luz e temperatura na germinação de sementes de quatro espécies de plantas daninhas do gênero Digitaria. Revista Brasileira de Sementes, Piracicaba, v.32, n.1, 2010.

REHDER, V. L. G.; SARTORATTO, A.; RODRIGUES, M. V. N.. Essential oils composition from leaves, inflorescences and seeds of Mikania laevigata Schultz Bip. ex Baker and Mikania glomerata Sprengel. Revista Brasileira de Plantas Medicinais, Botucatu, v.8, p.116-118, 2006.

SÁNCHEZ-MUÑOZ, B. A.; AGUILAR, M. I.; KING-DÍAZ, B.; RIVERO, J. F.; LOTINA-HENNSEN, B.. The Sesquiterpenes $\beta$ Caryophyllene and Caryophyllene Oxide Isolated from Senecio salignus Act as Phytogrowth and Photosynthesis Inhibitors. Molecules, Cidade do México, v.17, p.1437-1447, 2012. DOI: http://doi.org.10.3390/molecules17021437

SANTOS, D. M. M.; PITELLI, A. R.; BANZATTO, D. A.. Efeitos de herbicidas nos teores de clorofilas de Spirodela punctata. Planta Daninha, Viçosa, v.17, n.2, p.175-182, 1999. DOI: http://doi.org.10.1590/S0100-83581999000200001

SANTOS, J. B.; CURY, J. P.. Picão-preto: uma planta daninha especial em solos tropicais. Planta daninha, Viçosa, v.29, p.1159-1171, 2011. DOI: http://doi.org.10.1590/S0100$\underline{83582011000500024}$

SCRIVANTI, L. R.. Allelopathic potential of Bothriochloa laguroides var. laguroides (DC.) Herter (Poaceae: Andropogoneae). Flora, Córdoba, v.205, p.302-305, 2010. DOI: http://doi.org.10.1016/i.flora.2009.12.005
SILVA JÚNIOR, A. A.; RITTER, M. R.; ZAMBONIM, F. M.; DESCHAMPS, F. C.; ACENCO F. A. T. C.; BERTOLDI, F. C.. Um novo ecótipo de Mikania glomerata Spreng. (Asteraceae) rico em óleo essencial no Sul do Brasil. Revista Fitos, Rio de Janeiro, v.9, n.1, p.1-72, 2015. DOI: http://doi.org.10.5935/2446-4775.20150002

SILVA, I. D.; TAKATSUKA, F. S.; ROCHA, M. R.; CUNHA, M. G.. Efeito do extrato de sucupira (Pterodon emarginatus Vog.) sobre o desenvolvimento de fungos e bactérias fitopatogênicos. Pesquisa Agropecuária Tropical, Viçosa, v.35, p.109-115, 2005.

SOUZA, C. S. M.. Alelopatia do extrato aquoso de folhas de aroeira na germinação de sementes de alface. Revista Verde, Mossoró, v.2, n.2, p.96-100, 2007.

VERDEGUER, M.; GARCÍA-RELLÁN, D.; BOIRA, H.; PÉREZ, E.; GANDOLFO, S.; BLÁZQUEZ, M. A.. Herbicidal activity of Peumus boldus and Drimys winterii essential oils from Chile. Molecules, Valência, v.16, n.1, p.403-11, 2011. DOI: http://doi.org.10.3390/molecules 16010403

ZHENG, G. Q.; KENNY, P. M.; LAM, L. K. T.. Sesquiterpenes from clove (Eugenia caryphyllata) as potential anticarcinogenic agents. Journal of Natural Products, Minneapolis, v.55, p.999-1003, 1992. DOI: http://doi.org.10.1021/np50085a029

ZHU, X. Z.; HAN, C. X.; GAO, T.; SHAO, H.. Chemical Composition, Phytotoxic and Antimicrobial Activities of the Essential Oil of Scutellaria strigillosa Hemsley. Journal of Essential Oil Bearing Plants, Pequim, v.19, p.664-670, 2016. DOI: http://doi.org.10.1080/0972060X.2014.1000389 